\title{
The Projection from Medial Geniculate to Field Al in Cat: Organization in the Isofrequency Dimension
}

\author{
S. Brandner and H. Redies \\ Department of Neurobiology, Max-Planck-Institute for Biophysical Chemistry, D-3400 Göttingen, Federal Republic of \\ Germany
}

The topography of the anatomical projection from isofrequency contours (IFCs) in auditory thalamus to IFCs in primary auditory cortex (field $\mathrm{Al}$ ) was investigated in the cat. In each experiment, a best-frequency map of Al was obtained with electrophysiological recording techniques. Then, different retrogradely transported tracers (HRP, fluorescent dyes) were introduced into Al. In some experiments, different parts (e.g., dorsal, central, and ventral) of a previously mapped IFC were injected, each part with a different tracer. In other experiments, 2 or 3 rows of tracer injections were made at different dorsoventral levels of Al, over a large frequency range (5-38 $\mathrm{kHz}$ ); each injection row was oriented orthogonal to the IFCs and contained a different tracer.

The main mass of the labeled thalamic cells was found in the ventral nucleus of the medial geniculate body (MGv). The MGv cells projecting to a limited sector (1-2 $\mathrm{mm}$ in length in most experiments) of an IFC in Al form one or several densely packed neuron clusters of variable shape. The cells labeled by a given tracer are largely separated in space from cells labeled by a different tracer. Thus, different sectors of a cortical IFC receive input from different portions of the corresponding thalamic IFC. As a general rule, cells labeled from dorsal (ventral) injections are centered rostrally (caudally) in the part of MGv innervating Al. However, the topographic details are variable between individuals, and the rostrocaudal gradient is complicated by numerous irregularities and gradients.

Previous studies of the auditory thalamocortical projection in the cat have not recognized the topographic order in the isofrequency dimension. Instead, it was believed that different sectors of a cortical IFC were innervated by coincident thalamic populations.

The tonotopic fields of the mammalian auditory cortex receive most of their diencephalic afferents from the tonotopic nuclei of the auditory thalamus. For example, in the cat primary auditory cortex, (field AI) is innervated mainly by the ventral nucleus of the medial geniculate body (MGv; see Imig and Morel, 1983, for review). Cortical isofrequency contours (IFCs) with a given best frequency (BF) receive input from thalamic IFCs

\footnotetext{
Received Mar. 22, 1989; revised May 31, 1989; accepted June 26, 1989.

We thank Prof. Dr. Otto Creutzfeldt for his full support during this study, and Drs. Barry Lee and Paul Martin for improving the language. Susanne Lausmann, Eva Niksch, and Ursula Steveling provided excellent histological assistance.

Correspondence should be addressed to Dr. S. Brandner, Max-Planck-Institute for Biophysical Chemistry, Department of Neurobiology, Am Fassberg, D-3400 Göttingen, Federal Republic of Germany.

Copyright (C) 1990 Society for Neuroscience $0270-6474 / 90 / 010050-12 \$ 02.00 / 0$
}

of the same BF. Correspondingly, the anatomical projection from tonotopic thalamus to tonotopic cortex is characterized by a topographic gradient along the frequency axis (Andersen et al., 1980; Middlebrooks and Zook, 1983; Imig and Morel, 1984).

The main subject of the present paper is the structure of the thalamocortical projection in the isofrequency dimension. Is there also a topographic gradient along the isofrequency axis? This question has been investigated in 2 species in the past and has received different answers.

Andersen et al. (1980), working on the cat, injected tracer substances into electrophysiologically identified loci of field AI and found that the cells in MGv projecting to a locus in AI form a dorsoventrally oriented, folded sheet. After injection of small quantities of tracer into medium or high $\mathrm{BF}$ sites, this sheet exhibits marked discontinuities: it appears as a sequence of 4 6 patches arranged from dorsal to ventral, with little or no label between the patches (see Andersen et al., 1980, figure 7). Larger injections of tracer (diameter of injection site more than $1 \mathrm{~mm}$, according to Merzenich et al., 1982), into the same IFC, result in a sheet of similar size and dimensions as produced by a small injection, but with fewer or no discontinuities. The authors suggested that these findings could be explained by the hypothesis that there are alternating binaural bands in $\mathrm{MGv}$, as in the cortex. ${ }^{1}$ A small injection of tracer into AI, restricted to 1 binaural band (e.g., an EI-band), would label binaural bands of only one type in MGv (namely the thalamic EI-bands) and thus result in discontinuous labeling. A larger injection, covering an EIand an EE-band, would label EI- and EE-bands in MGv and thus produce a continuous sheet. The observation that the size of the labeled thalamic sheet is largely independent of the size of the injection site suggested that the projection from a thalamic to a cortical IFC is structured as schematically shown in Figure $1 a$ : All thalamic bands of 1 binaural class would project to each cortical band of the same class. There is thus a high degree of divergence/convergence in the projection; i.e., in a cortical IFC, different loci of the same binaural class receive afferents from coincident thalamic populations (Andersen et al., 1980; Merzenich et al., 1982).

Middlebrooks and Zook (1983), also working on the cat, have basically supported this model, with one modification. They mapped binaural bands in field $\mathrm{AI}$ and then injected tracer into

\footnotetext{
There are 2 types of binaural bands in AI: neurons in the excitatory/excitatory bands ("EE-bands") are excited by acoustic signals applied to the contralatera and to the ipsilateral ear. In the excitatory/inhibitory bands ("EI-bands"), the cells are excited by contralateral stimuli, but inhibited by simultaneously applied ipsilateral stimuli (Imig and Adrian, 1977; Middlebrooks et al., 1980; but see also Phillips and Irvine, 1979).
} 
an identified EE- and/or EI-domain. The authors concluded that different cortical EI-bands are probably innervated by coincident thalamic populations; the thalamic arrays projecting to different EE-bands, on the other hand, coincide largely, but not completely (see original paper for more details).

Thus, according to these studies on the cat, the projection from a thalamic to a cortical IFC has no simple, spatial structure. Rather, a functional factor, namely binaural information processing, seems to determine the connectional pattern in the isofrequency dimension. This conclusion, however, is in contrast to recent findings from our laboratory on the auditory thalamocortical projection in the guinea pig (Redies et al., 1989b). We mapped cortical IFCs and then injected retrograde tracers into different portions of an identified IFC. We found that there was a straightforward spatial gradient in the isofrequency dimension: the dorsal portion of a cortical IFC was innervated by the caudolateral part of the corresponding thalamic IFC, while more ventral portions of the cortical IFC received afferents from more rostromedial parts of the thalamic IFC. The "divergence/ convergence" architecture (Fig. 1a) described in the cat was not observed. Rather, the projection can be described as a "pointto-point" connection (Fig. 1b) (Redies et al., 1989b).

Do the apparent differences between cat and guinea pig really exist? And if so, what is their functional significance? With these questions in mind, we reinvestigated the connection between thalamic and cortical IFCs in cat. In each experiment, a BF map of field AI was obtained with microelectrode mapping techniques. Then, different retrograde tracers were injected into different parts of electrophysiologically identified cortical IFCs. Subsequent analysis of the spatial distribution of labeled cell clusters in MGv revealed a topographic organization along the isofrcquency axis and showed that the "divergence/convergence" model (Fig. 1a) is not valid for the cat.

\section{Materials and Methods}

Animals, surgery, and anesthesia. Adult cats (body weight $2.5-4 \mathrm{~kg}$ ) without signs of middle ear disease were used. The animals were initially anesthetized with sodium pentobarbital $(35 \mathrm{mg} / \mathrm{kg})$ or a combination of xylazine hydrochloride $(2 \mathrm{mg} / \mathrm{kg})$ and ketamine hydrochloride (15 $\mathrm{mg} / \mathrm{kg})$. In addition, atropine sulfate $(0.5 \mathrm{mg} / \mathrm{kg})$ was given to inhibit tracheal secretion and prednisolone-21-hemisuccinate-sodium $(4 \mathrm{mg} /$ $\mathrm{kg}$ ) to prevent brain edema. A catheter was introduced into the saphenous vein and the trachea was cannulated. The animal was then relaxed with an initial dose of $10 \mathrm{mg} / \mathrm{kg}$ gallamine triethiodide and artificially respirated with a gas mixture of $80 \% \mathrm{~N}_{2} \mathrm{O}, 19 \% \mathrm{O}_{2}$, and $1 \%$ $\mathrm{CO}_{2}$. End-expiratory $\mathrm{CO}_{2}$ level was monitored and maintained at 3.5$4 \%$, and body temperature was kept near $38^{\circ} \mathrm{C}$. The electrocardiogram was continuously monitored on an oscilloscope. During the mapping experiment, a solution containing gallamine triethiodide $(10 \mathrm{mg} / \mathrm{kg} / \mathrm{hr})$, glucose $(40 \mathrm{mg} / \mathrm{kg} / \mathrm{hr})$, and sodium pentobarbital $(1 \mathrm{mg} / \mathrm{kg} / \mathrm{hr})$, dissolved in Ringer's solution, was continuously administered.

The animal's head was rigidly fixed without use of earbars. The skull overlying field AI was removed and the dura carefully resected. The cortex was then covered with warm agar-agar (3\% in Ringer's solution) to reduce brain pulsations.

Acoustic stimuli and frequency tuning curves. Acoustic stimuli were generated in a table lookup procedure by a PDP-11/73 computer extended with a TZQ-1 1 signal-coprocessor and a 16-bit D/A converter. The converted signals were presented to the animal via a free-field loudspeaker (Multicel), which was positioned at a distance of $70 \mathrm{~cm}$ from the animal's head, contralateral to the side where the cortex was exposed, at $45^{\circ}$ from the sagittal plane in the anterior hemifield.

Frequency tuning curves were obtained by recording neuronal responses to trapezoideally shaped pure tones (duration: $100 \mathrm{msec}$, rise/ falltime: $8 \mathrm{msec}$ ). For each tuning curve, tones of 15-20 different frequencies ranging from 0.5 to 32 or $38 \mathrm{kHz}$, with interfrequency intervals of $0.25-0.5$ octave, were generated by the computer. Each frequency
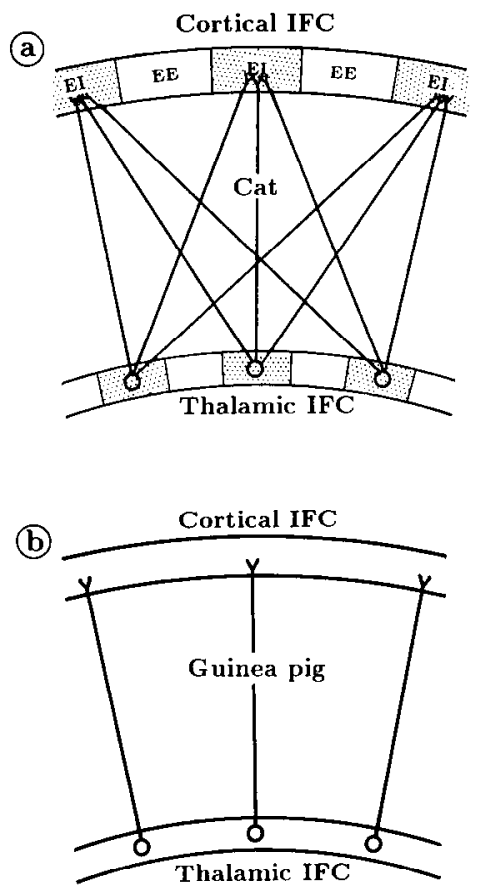

Figure 1. a, Schematic representation of the "divergence/convergence" architecture in the isofrequency dimension, as suggested for cat. The lines between the thalamic and the cortical IFCs represent the anatomical connections between both structures; for clarity, only the EI-part of the projection is shown. $b$, Schematic representation of the "point-to-point" architecture, as described in guinea pig. Adopted from Redies et al. (1989b). See text for further explanation.

was emitted in 6-9 different amplitudes between $0 \mathrm{~dB}$ and $80 \mathrm{~dB}$ SPL. A tone of a given frequency/amplitude combination was repeated 3-5 times, and the order of the signals was randomized. See Redies et al. (1989a) for more details. All experiments were conducted in a soundproof room.

The acoustic system was calibrated as follows: before the experiment, a condenser microphone ( $1 / 4$ in., Brüel \& Kjaer) was placed between the animal's ears. Then, for all stimulation frequencies, the sound pressure level for a fixed amplitude of the electric signal was measured by the microphone. The resulting frequency-transfer-function was fed into the computer. During the experiment, the signal amplitude was adjusted by a computer-controlled attenuator to achieve equal sound pressure for all frequencies.

Mapping experiments. Glass-coated metal microelectrodes ("Elgiloy" electrodes; Suzuki and Azuma, 1976), with impedances of 1.5-5 M $\Omega$ at $1000 \mathrm{~Hz}$, were used to record action potentials from clusters of neurons or single cells. The electrode was introduced into the cortex by means of a hydraulic microdrive, with an orientation approximately perpendicular to the cortical surface. Tuning curves were recorded from those cortical layers where cells responded best to tonal stimuli. In AI, this was normally 500-1500 $\mu \mathrm{m}$ below the pial surface, except near the anterior or posterior ectosylvian sulcus, where the electrode had to be advanced deeper into the cortex to find responsive cells. See Redies et al. (1989a) for other details of data acquisition and storage.

In each mapping experiment, tuning curves from 20-80 electrode penetrations were obtained, with recordings from 1-3 different neurons or clusters of neurons per penetration. In many cases, we also investigated neuronal responses to various complex acoustic stimuli, but these data will not be presented here. The recording sites were marked on a photograph of the cortical surface, using the surface vasculature for positional reference. The transition between $\mathrm{AI}$ and AII, i.e., the ventral border of $\mathrm{AI}$, was identified by the broader frequency tuning curves in AII, and by sudden changes in BF that did not fit into the AI tonotopy. In the auditory cortex dorsal to AI, responsiveness to pure tones is weaker than in AI, and often, cells are not driven by tonal stimuli at all. In some animals, cells with high BFs (above $10-15 \mathrm{kHz}$ ) predominate 


\begin{tabular}{|c|c|c|c|}
\hline Animal & Tracer & Injection site & Figure \\
\hline \multirow[t]{2}{*}{ САT006 } & $4 \mathrm{NY}$ & $5.7-23 \mathrm{kHz}$, dorsal & - \\
\hline & $6 \mathrm{Bb}$ & $5.7-23 \mathrm{kHz}$, ventral & \\
\hline \multirow[t]{3}{*}{ CAT010 } & $3 \mathrm{NY}$ & $5.7-38 \mathrm{kHz}$, dorsal & \\
\hline & $5 \mathrm{HRP}$ & $5.7-38 \mathrm{kHz}$, central & 2 \\
\hline & $5 \mathrm{Bb}$ & $5.7-38 \mathrm{kHz}$, ventral & \\
\hline \multirow[t]{2}{*}{ CAT012 } & $1 \mathrm{NY}$ & $11 \mathrm{kHz}($ see text) & - \\
\hline & $1 \mathrm{Bb}$ & $11 \mathrm{kHz}$ & \\
\hline \multirow[t]{2}{*}{ CAT016 } & $2 \mathrm{NY}$ & 4-6.7 kHz, dorsal & 6 \\
\hline & $4 \mathrm{Bb}$ & $4-6.7 \mathrm{kHz}$, center, ventral & \\
\hline \multirow[t]{3}{*}{ САT017 } & $2 \mathrm{NY}$ & $4-6.7 \mathrm{kHz}$, dorsal & \\
\hline & 3 HRP & $4-6.7 \mathrm{kHz}$, center & - \\
\hline & $2 \mathrm{Bb}$ & $4-6.7 \mathrm{kHz}$, ventral & \\
\hline \multirow[t]{2}{*}{ CAT018 } & $4 \mathrm{Bb}$ & $4-6.7 \mathrm{kHz}$, dorsal & 7 \\
\hline & $3 \mathrm{NY}$ & $4-6.7 \mathrm{kHz}$, ventral & \\
\hline \multirow[t]{3}{*}{ CAT020 } & $3 \mathrm{Bb}$ & $16-32 \mathrm{kHz}$, dorsal & \\
\hline & 3 HRP & $16-32 \mathrm{kHz}$, center & 5 \\
\hline & $1 \mathrm{NY}$ & $16-32 \mathrm{kHz}$, ventral & \\
\hline \multirow[t]{2}{*}{ САТ026 } & $3 \mathrm{Bb}$ & $11 \mathrm{kHz}$, dorsal & - \\
\hline & $1 \mathrm{NY}$ & $11 \mathrm{kHz}$, ventral & \\
\hline \multirow[t]{2}{*}{ СAT027 } & 4 HRP & $19-27 \mathrm{kHz}$, dorsal & - \\
\hline & $3 \mathrm{Bb}$ & $19-27 \mathrm{kHz}$, center & \\
\hline \multirow[t]{3}{*}{ САТ033 } & $3 \mathrm{Bb}$ & 19-32 kHz, dorsal & \\
\hline & 3 HRP & $19-32 \mathrm{kHz}$, central & 3,4 \\
\hline & $2 \mathrm{NY}$ & $19-32 \mathrm{kHz}$, ventral & \\
\hline
\end{tabular}

in this region. Thus, responsiveness to pure tones, and changes in tonotopy, were used as criteria to determine the dorsal border of AI. Duration of the electrophysiological experiments was 20-35 hr.

Tracer injection and survival time. HRP (Sigma VI, St. Louis, MO) and the fluorescent dyes Bisbenzimid (Bb) (Riedel de Haen AG, Seelze Hannover, FRG) and nuclear yellow (NY) (Dr. Loewe, Hoechst AG, Frankfurt-M., FRG) were used as retrogradely transported tracer substances (Kuypers et al., 1979; Bentivoglio et al., 1980; Mcsulam, 1982). In each experiment, 2 or 3 different tracers were introduced into electrophysiologically identified cortical sites. HRP and $\mathrm{Bb}$ were implanted in cristalline form (Mori et al., 1980; Redies et al., 1989b); the hollow tips of the implantation pipettes, which contained the tracer, measured $50-80 \mu \mathrm{m}$ in length and had an inner diameter of $40-70 \mu \mathrm{m}$. NY $(2 \%$ suspension in aqua dest.) was injected through a glass pipette (outer diameter $80-120 \mu \mathrm{m}$ ) mounted on a $1-\mu \mathrm{l}$ Hamilton syringe. NY injection volumes varied from 0.05 to $0.1 \mu \mathrm{l}$.

Perfusion, histochemistry. Tracer injection was followed by a survival time of 24-30 hr. During this period, artificial respiration was with room air. A solution containing an augmented dose of sodium pentobarbital $(4 \mathrm{mg} / \mathrm{kg} / \mathrm{hr})$ and gallamine triethiodide, glucose, and Ringer's in concentrations as described above was continuously administered. After the survival time, the animal was given an additional dose of sodium pentobarbital and transcardially perfused with $0.9 \%$ saline, followed by fixative ( $1 \%$ paraformaldehyde, $1.25 \%$ glutaraldehyde in phosphate buffer), $10 \%$ buffered sucrose, and $20 \%$ buffered sucrose. The brain was then removed from the skull and cut on a freezing microtome (frontal sections, $50 \mu \mathrm{m}$ thick). Every second section was immediately mounted to minimize diffusion of the fluorescent dyes from the injection sites and labeled neurons. This series of sections was used later for analysis of $\mathrm{NY}$ and $\mathrm{Bb}$ cells. The remaining sections were reacted for HRP, following the tetramethyl benzidine (TMB) protocol of Mesulam (1982, see appendix III, pp. 127-130, for details).

Data evaluation. The caudal thalamus was carefully checked for occurrence of labeled cells using light and fluorescence microscopy. Labeled cells were marked on a drawing of the outlines of the section by means of an $X / Y$ plotter coupled to the microscope. In the sections that were not TMB-processed, Bb- or NY-labeled neurons were characterized by a fluorescent nucleus and by a ring of brilliant fluorescent elements surrounding the nucleolus. Labeled cells without the nucleolar ring were also observed, but not included in analysis, as they were probably glial cells (Kuypers et al., 1979, 1980).

The location of cells labeled by Bb or NY relative to the HRP-labeled cells was determined by superimposing tracings of adjacent sections from the TMB-processed series and the nonprocessed series, and by direct comparison of the HRP-labeled cells and the fluorescent cells in the same, TMB-processed sections. The appearance of the fluorescent cells had changed in the TMB-processed sections, in that the characteristic fluorescent ring around the nucleolus had disappeared in many cells. Thus, there was no reliable criterion to distinguish between labeled neurons and glia in the TMB-processed sections. However, glial cells can only uptake label if they are located in the vicinity of retrogradely labeled neurons, and in our material, the spatial distribution of the fluorescent cells in the TMB-processed sections was always very similar to that in the adjacent, not TMB-processed sections. Thus, the possible labeling of glial cells does not alter the topographic features of the TMBprocessed sections.

\section{Results}

The tonotopic organization of AI, and the thalamic nuclei projecting to this field, are treated in detail in the literature (for review see Imig and Morel, 1983; Brugge, 1985; Brugge and Reale, 1985; Winer, 1985). Our data confirm these results. Therefore, in the following, we describe the topographic organization of the projection only. In describing the medial geniculate body, we follow the scheme proposed by Morest (1964, 1965).

Successful experiments with injections into AI were carried out in 10 animals. In Table 1, the experiments are listed in chronological order, with the tracer substances used and the location of the injection sites.

The apparent HRP-injection sites, as defined by the zone around the injection site center where dense tracer deposit was visible, had diameters between 0.5 and $1.3 \mathrm{~mm}$. However, the effective injection sites, i.e., the cortical areas in which tracer uptake really occurs, are not necessarily of the same size (for review of this methodological issue, see Mesulam, 1982; Fahrbach et al., 1984). The effective Bb-injection sites had, presumably, a size similar to the effective HRP-injection sites, since retrograde transport was quantitatively similar. For NY, the area of effective uptake was either the same, or, in some cases, slightly larger, than for $\mathrm{HRP}$ and $\mathrm{Bb}$, as suggested by a similar or greater number of retrogradely NY-labeled cells (see below).

\section{Topography along the isofrequency axis}

\section{Overview experiments}

In 2 experiments, rows of tracer injections were made at different dorsoventral levels of AI, covering a large frequency range; each row was oriented approximately orthogonal to the IFCs and contained a different tracer (see Fig. $2 a$ ). The purpose was to determine whether there is a topographic order at all in the isofrequency dimension, and, if so, to obtain a preliminary idea of its layout.

The BF map of case CAT010 is shown in Figure $2 a$. Three injections of NY were made into the dorsal portion of AI (filled circles in Fig. $2 a$ ), into sites with BFs between 5.7 and $38 \mathrm{kHz}$; IIRP was injected into 5 locations (crossed circles) situated ca. $1.2 \mathrm{~mm}$ ventral of the $\mathrm{NY}$-injections, and $\mathrm{Bb}$ was introduced into 5 sites (open circles) about $1.8-2 \mathrm{~mm}$ ventral to the HRP. injections.

Figure $2 b$ comprises drawings of frontal sections through the auditory thalamus and shows the position of the labeled cells, represented by dots, relative to other brain structures. The results are illustrated in greater detail in Figure $2 c$, where the 
differently labeled cells are represented by different symbols (see below). The section outlines and other anatomical landmarks are omitted in Figure 2c, but the location of the cells can be inferred by comparing with Figure $2 b$. In the insets, the distance of each section to the first (rostral) section shown is given.

The labeled cells in the auditory thalamus are organized in 2 distinct columns: one array (named "medial column," MC) extends through the lateral part of posterior thalamus, the deep dorsal nucleus of MG, and the rostral part of the medial nucleus of MG. A second array ("lateral column," LC) is situated in MGv (Andersen et al., 1980). Rostrally, LC is a dorsoventrally oriented, more or less continuous band (Fig. 2c, sections $71-$ 55); caudally, it diverges into a large dorsal component ( $\mathrm{LCd}$ ) and a smaller ventral component (LCv) (sections 51-39). See Figure $2 \mathrm{C}$, section 43 , for clarification of the above defined abbreviations. The $\mathrm{LCd}$ is located in the lateral and transitional parts of MGv, whereas the $\mathrm{LCv}$ is partly or entirely located in pars ovoidea of the MGv, as defined by Morest $(1964,1965)$.

In the following, cells labeled from dorsal injections are designated as di-cells (filled circles in all figures), cells labeled from medial injections as mi-cells (crossed circles), and those labeled from ventral injections as vi-cells (open circles). It is immediately clear from Figure $2 c$ that in LC, the cells labeled by different tracers are not randomly intermingled. Most of the di-cells are situated rostrally, while the bulk of the vi-cells is located caudally. The mi-cells are most abundant in the intermediate sections. This rostrocaudal gradient is also apparent in Figure $2 d$ : the graph shows the percentage of cells labeled by each tracer as a function of the section's position on the rostrocaudal axis. Furthermore, differently labeled cells occurring in the same section are spatially separate; i.e., cells of the same label are grouped together in 1 or several clusters, with no or only slight overlap between differently labeled clusters (Fig. $2 c$ ).

In $\mathrm{MC}$ also, a tendency for segregation into clusters of cells, labeled by the same tracer, is evident in most, but not all, sections.

In a further overview experiment (CAT006, not illustrated), $4 \mathrm{NY}$-injections were placed dorsally in $\mathrm{AI}$, over a frequency range from 6 to $22 \mathrm{kHz}$, and $6 \mathrm{Bb}$-injections wcrc positioned about $1.7 \mathrm{~mm}$ ventral to the NY-injections. Rostrally in LC, the di-cells (NY) predominated, while caudally, vi-cells were most abundant. The differently labeled cells in LC formed different clusters that overlapped only slightly or not at all. Thus, results were similar as in CAT010, though some differences in the details of topography were also noted. In MC of CAT006, mainly di-cells were found.

The overview experiments suggest that the projection from $\mathrm{MGv}$ to $\mathrm{AI}$ is topographically organized in the isofrequency dimension. The differently labeled cells are spatially segregated, and there is a tendency for di-cells to be located rostrally and for vi-cells to be located caudally. We have further examined the topography of the projection to single cortical IFCs. The results are presented separately for the high-frequency (11-38 $\mathrm{kHz})$ and the medium-frequency parts $(3-8 \mathrm{kHz})$ of the projection. The low-frcquency part was not investigated, since the cortical IFCs for BFs below $3 \mathrm{kHz}$ run partly in the rostral bank of the posterior ectosylvian sulcus in most animals and are difficult to access.

\section{Topography of the high-frequency projection}

In experiment CAT033, $3 \mathrm{Bb}$-injections were made dorsally, 3 HRP-injections centrally, and $3 \mathrm{NY}$-injections $(0.05 \mu \mathrm{l})$ ven- trally into the $22-32-\mathrm{kHz}$ IFC (Fig. 3a). The main mass of the di-cells $(\mathrm{Bb}$, filled circles in Fig. $3 b)$ is situated in the anterior half of LC. In the rostralmost sections (69-57), the di-array has the form of an elongated band running parallel to the dorsolateral margin of the MG. Further caudally (section 53), the band divides into a dorsal part (LCd) and a ventral part (LCV). The di-cells in LCv form a compact group, whereas those in LCd diverge into 2-3 clusters. The mi-cells (HRP, crossed circles in Fig. $3 b$ ) form a continuous column rostrally and diverge into a dorsal and a ventral component caudally. The roughly crescentlike shape of the mi-array is depicted, in a parasagittal perspective, in Figure $3 c$. Most of the vi-cells (NY, open circles in Fig. $3 b$ ) are situated in the caudal half of LC. They form several distinct columns that are not continuous or only loosely coherent. Two vi-columns, a dorsal and a ventral, are situated in LCd. These are connected to each other by a thin cell bridge in some sections (arrow in section 49). A third band of vi-cells, not continuous with the former 2 columns, is located in LCv (sections 53-37). See Figure $3 c$ for a schematic drawing of these results.

Section 41 (see Fig. $3 b$ ) was chosen as an example to illustrate the topography in the caudal half of LC in all detail. Figure $4 a$ is a photomicrograph of the NY-labclcd fluorcscent cells; Figure $4 b$ is a photomicrograph of the HRP-cells. Both photographs were taken from the same, TMB-processed section, and, therefore, the fluorescent elements are possibly in part glial cells (Bentivoglio et al., 1980; Kuypers et al., 1980; see also Materials and Methods). Figure $4 c$ is a superimposed tracing of both photographs. The arrows from $c$ to $b$ and from $b$ to $a$ indicate corresponding points in the figures. The NY-cells (ventral injection) and the HRP-cells (medial injection) form the following sequence of alternating clusters (the numbers corresponding to the circled numbers in Fig. 4c): (1) dorsally, an NY-group (vicells); (2) further ventrally, an HRP-group (mi-cells); (3) ventromedially, another NY-group (vi-cells); (4) a gap separating LCd from LCv; (5) dorsomedially in LCv, an NY-group (vicells); (6) then, a small HRP-group (mi-cells); and (7) ventrolaterally, again an NY-group (vi-cells).

There is some overlap between the dorsal HRP-group (2) (circled number in Fig. 4c) and the neighboring NY-groups (1) and (3), in that the cells in the border regions of these groups partly intermingle. However, the bulk of the HRP-cells of group 2 lies in an area almost entirely void of NY-cells, and vice versa. In LCV (groups 5-7), only 1 of 7 HRP-cells lies in an NY-region. A few HRP-cells occur apart from the main HRP-clusters (2 and 6) and are apparently randomly distributed; some lie amid the NY-cells, others in isolated positions. In other sections of caudal LC of CAT033, overlap was about the same as in section 41 , or less.

The topography in rostral LC is easier to describe. The micells are located medial and caudal to the di-cells. Slight overlap between the di- and mi-cells was observed. The few vi-cells in rostral LC are situated medially of the mi- and di-cells (section 57) and are not intermingled with the other clusters at all.

In summary, the differently labeled cells in CAT033 are largely separated in space. There is virtually no overlap between the di- and vi-cells. The partial overlap of the mi-cells with the viand di-cells could be due to the small distance between the injection sites: the ventral Bb-injection site and the dorsal HRPinjection site (see Fig. $3 a$ ) are separated by only about $0.7 \mathrm{~mm}$ (measured from center to center), and overlap of the effective injection sites in the cortex cannot be excluded (see Discussion). 


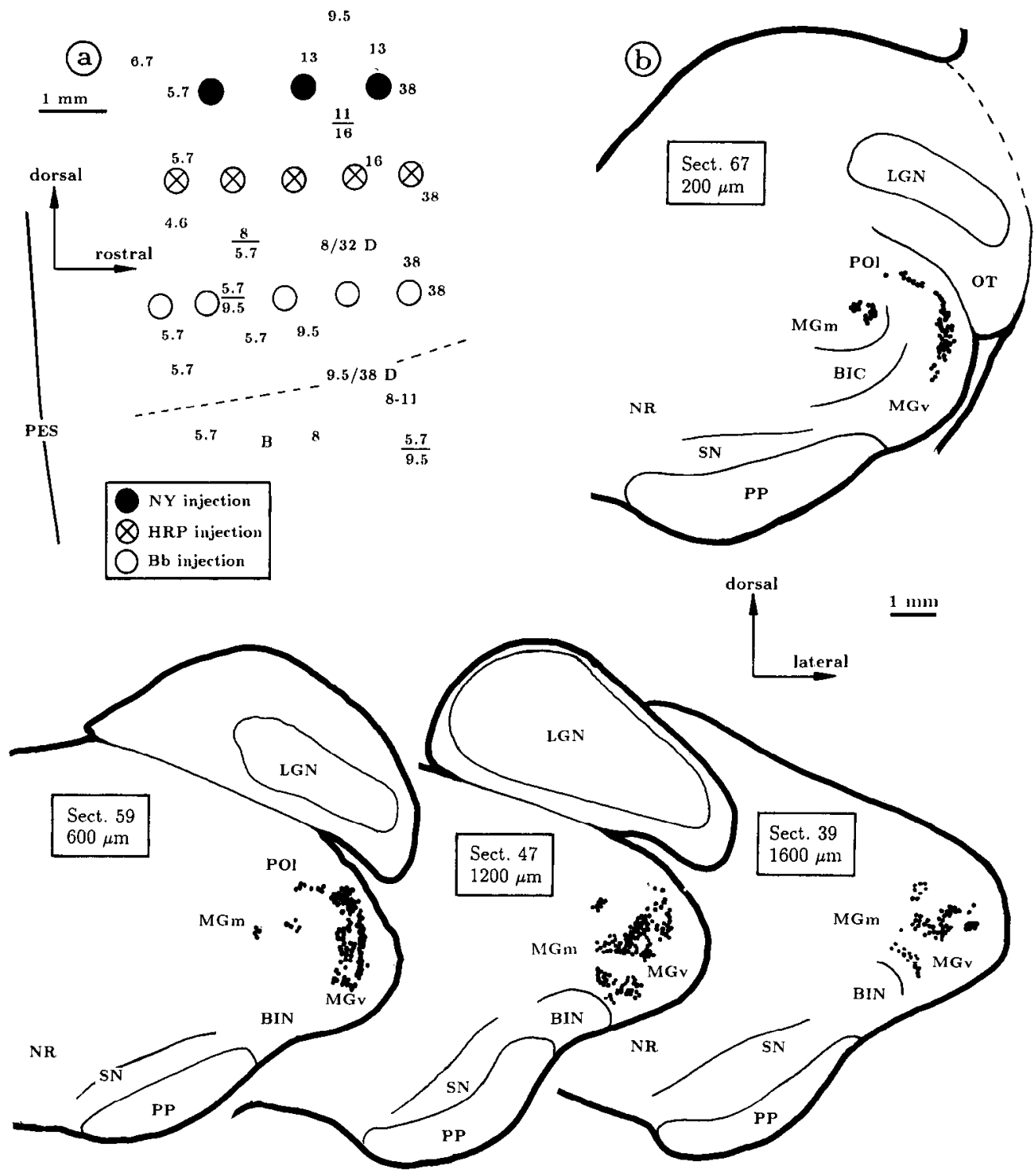

Figure 2. Experiment CAT010. a, BF map of field AI. Each recording site is represented by a number or a letter. The numbers show BFs in kHz; numbers separated by an oblique slash and followed by $D$ (e.g., $8 / 32 D$ ) stand for double-peaked tuning curves with 2 response maxima. Numbers separated by a horizontal line indicate that 2 different BFs were recorded, from 2 different cells (or cell clusters), in this penetration (see Materials and Methods). $B$ symbolizes a broad tuning curve without clear BF, and $N$ means no response to pure tones. The tracer injection sites are represented by circles (see inset). For orientation, the posterior ectosylvian sulcus $(P E S)$ is indicated by a solid line, and the approximate ventral border of AI by a broken line. $b$. Drawings of frontal sections through caudal thalamus. Section 67 (upper right) is from the rostral quarter of the labeled cell column in MGv, and section 39 (lower right) from the caudal quarter. The 2 other sections are from intermediate levels (the distance indications in the insets correspond to those in Fig. $2 c$, see below). Retrogradely labeled cells are represented by dots (no distinction is made here between cells labeled by different tracers). $c$, Spatial distribution of the differently labeled thalamic cells (frontal sections). Each circle stands for 3-10 labeled

The same holds for the distance between the HRP- and the NYinjections, about $1 \mathrm{~mm}$ (Fig. $3 a$ ).

The MC of CAT033 has a similar appearance as in the overview experiments, with a tendency for different labeled cells to form distinct cell groups.

In experiment CAT020, Bb was injected dorsally, HRP centrally, and NY ventrally into the $16-32-\mathrm{kHz}$ IFC in field AI (Fig. 5a). Though the injections were placed in similar locations as in CAT033, the results (Fig. 5, $b, c$ ) differed in several respects. The rostrocaudal gradient is stronger in CAT033 than in CAT020 (compare Figs. 3c, 5c). Moreover, in CAT033, the di-cells and the mi-cells each formed one continuous array. In CAT020, the corresponding cell populations werc not continuous: in all scc- tions, there were (for each tracer) at least 2 clusters, a dorsal and a ventral. In CAT020, in the rostral part of LC, the miand vi-cells occurred laterally of the di-cells (Fig. $5 b$ ). In CAT033 (Fig. 3b, sections 65-53), the vi- and mi-cells were situated medially of the di-cells. The structure of $\mathrm{LCV}$ is more similar in both animals, at least in its rostral portion (CAT020: section 64 in Fig. 5b; CAT033: section 53 in Fig. $3 b$ ).

In experiment CAT026 (not illustrated), $3 \mathrm{Bb}$-injections were placed dorsally in AI, into the $11-\mathrm{kHz}$ IFC, and NY (1 injection) was introduced $3 \mathrm{~mm}$ further ventrally into the same IFC. The di-cells were situated rostrally in LC and consisted of a single, continuous array. The vi-cells were centered further caudally and formed 3 clusters that were connected by very thin cell 

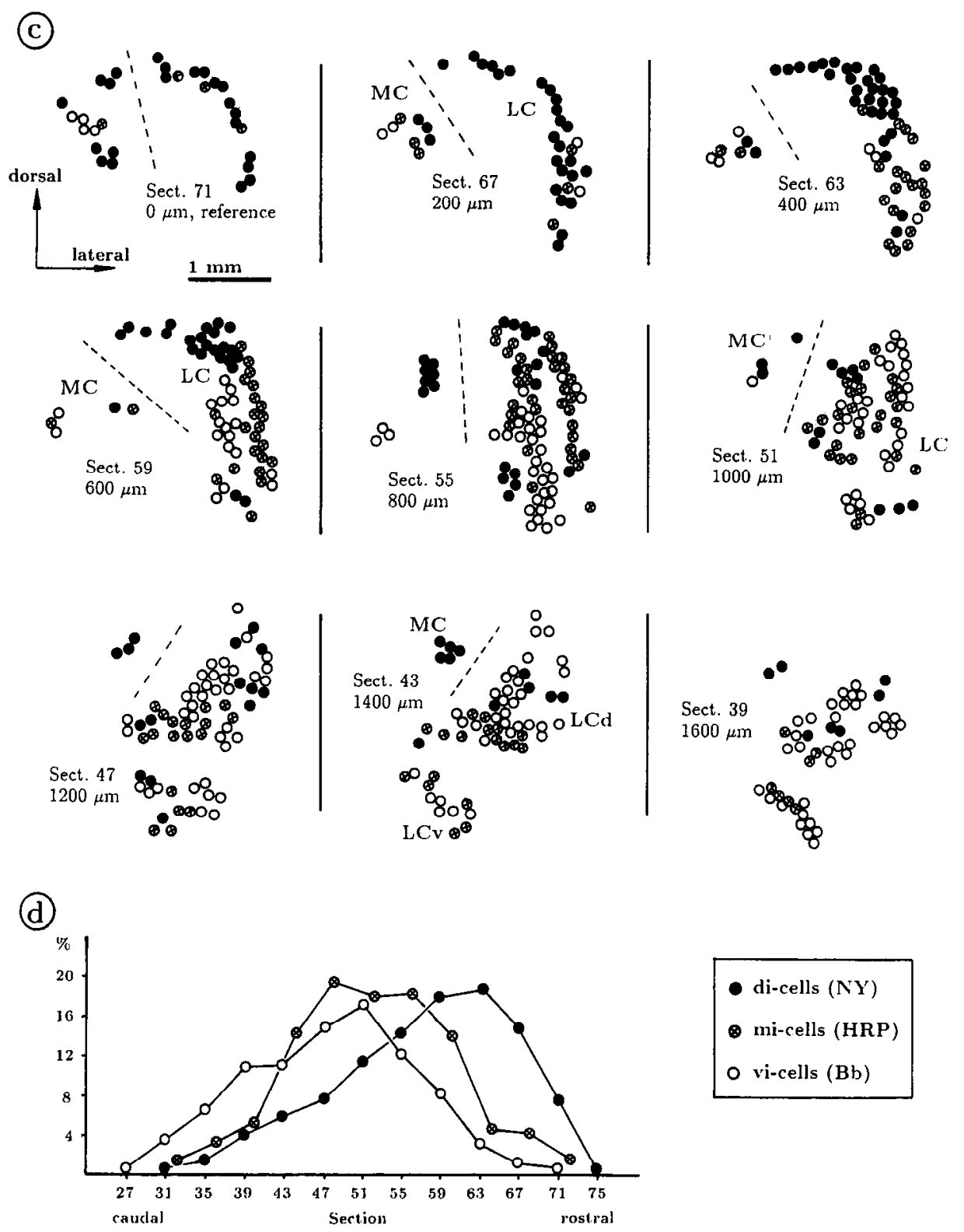

cells. The di-cells $(N Y)$ are represented by black circles, the mi-cells $(H R P)$ by crossed circles, and the vi-cells $(B b)$ by open circles. The most rostral section is 71 (upper left), the most caudal section is 39 (lower right). In the insets, the distance of each section to the most rostral section shown (reference) is given. MC is situated left and LC right of the broken lines. Sections 67, 59, 47, and 39 are also shown in b, and the position of the labeled arrays relative to other brain-stem structures can be inferred from there. $d$, Histogram of the rostrocaudal distribution of the differently labeled cells. In the abscissa, the position of each section on the rostrocaudal axis is given. The ordinate is the percentage of cells in LC labeled by a given tracer in the specific section, relative to all cells in LC labeled by this tracer. See text.

bridges only. In rostral LC, the di-cells were located lateral of the vi-cells, as in CAT033. In LCd, the di-cells were bordered by vi-cells dorsally and ventromedially, as in CAT020 and CAT033. There was virtually no overlap between the differently labeled populations.

In experiment CAT027 (not illustrated), HRP was injected dorsally ( 4 injections, spaced over $1 \mathrm{~mm}$ ) and $\mathrm{Bb} 0.5 \mathrm{~mm}$ further ventrally ( 3 injections, spaced over $1 \mathrm{~mm}$ ) into the $19-27-\mathrm{kHz}$ IFC in AI. The labeled cells were similarly distributed as the di- and mi-cells in CAT033 (Fig. 3c). Particularly, rostrally in LC of CAT027, the di-cells were situated laterally of the vicells; the spatial segregation of the differently labeled cells was also comparable to that of the di- and mi-cells in CAT033.
In experiment CAT012, an NY-injection was made into the $11-\mathrm{kHz}$ IFC, and a $\mathrm{Bb}$-injection $1.8 \mathrm{~mm}$ further ventrally, into the same IFC. The BF map obtained in this case was not detailed enough to determine the dorsoventral position of the injection sites. This case confirms the rostrocaudal gradient in LC, as described above, and the spatial segregation of differently labeled cells in MGv. Further evaluation of CAT012 was not carried out, because of the uncertainties in the location of the injection sites.

In conclusion, the high-frequency experiments confirm that different sectors of a cortical IFC are innervated by different parts of the thalamic IFC. When the injection sites of different tracers are separated by more than 1.5 or $2 \mathrm{~mm}$ in cortex, 

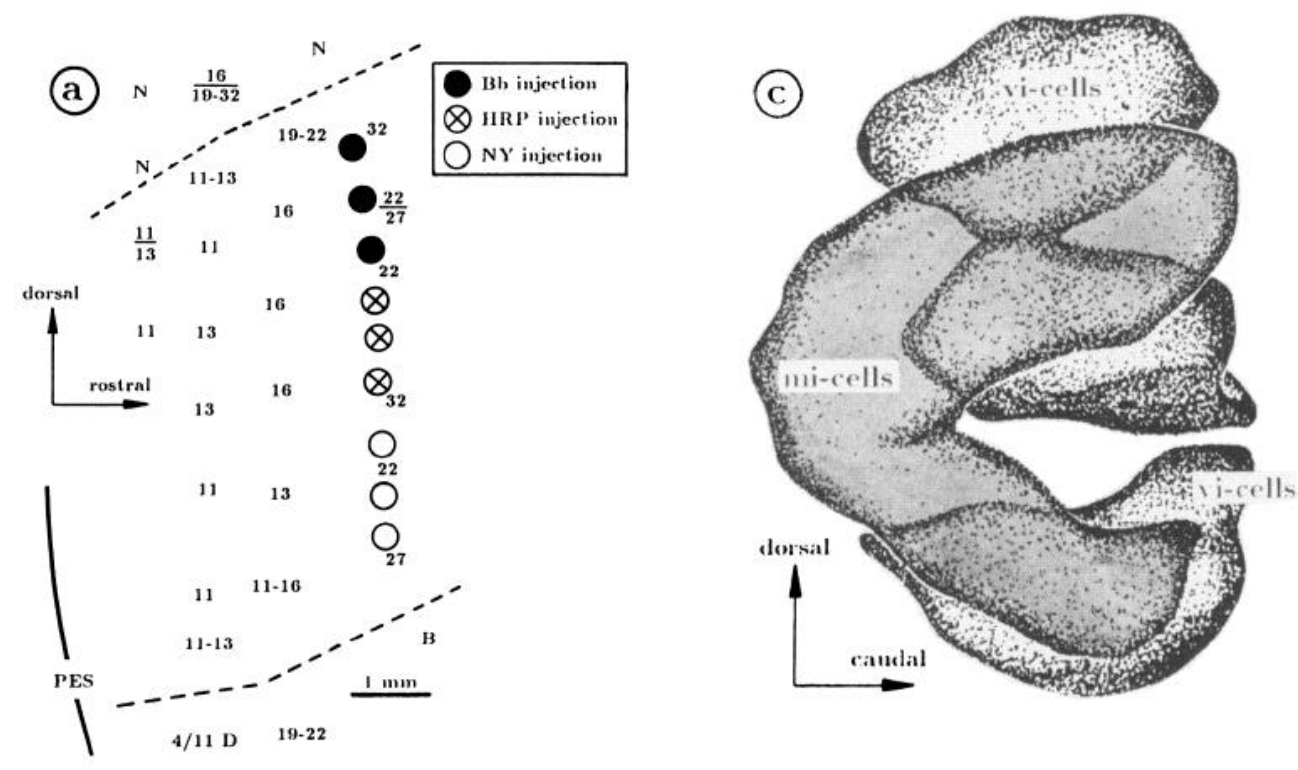

Figure 3. Experiment CAT033, $a, \mathrm{BF}$ map with injection sites. Broken lines indicate the approximate dorsal and ventral borders of AI. $b$, Drawings of the labeled cells. The arrow in section 49 points to a thin cell bridge that connects the dorsal and ventral vi-cell clusters in LCd (see text). The mi-cells (HRP, crossed circles) of some sections are shown in detail in Figure 4. For further explanation of $a$ and $b$, see Figure 2. $c$, Schematic representation of the $\mathrm{mi}-$ and vi-cells in $\mathrm{LC}$, in a parasagittal perspective, viewed laterally (for clarity, the di-cells were omitted). The drawing shows the main features of the labeled columns in CAT033; some details (e.g., slight overlap of differently labeled clusters) were neglected.
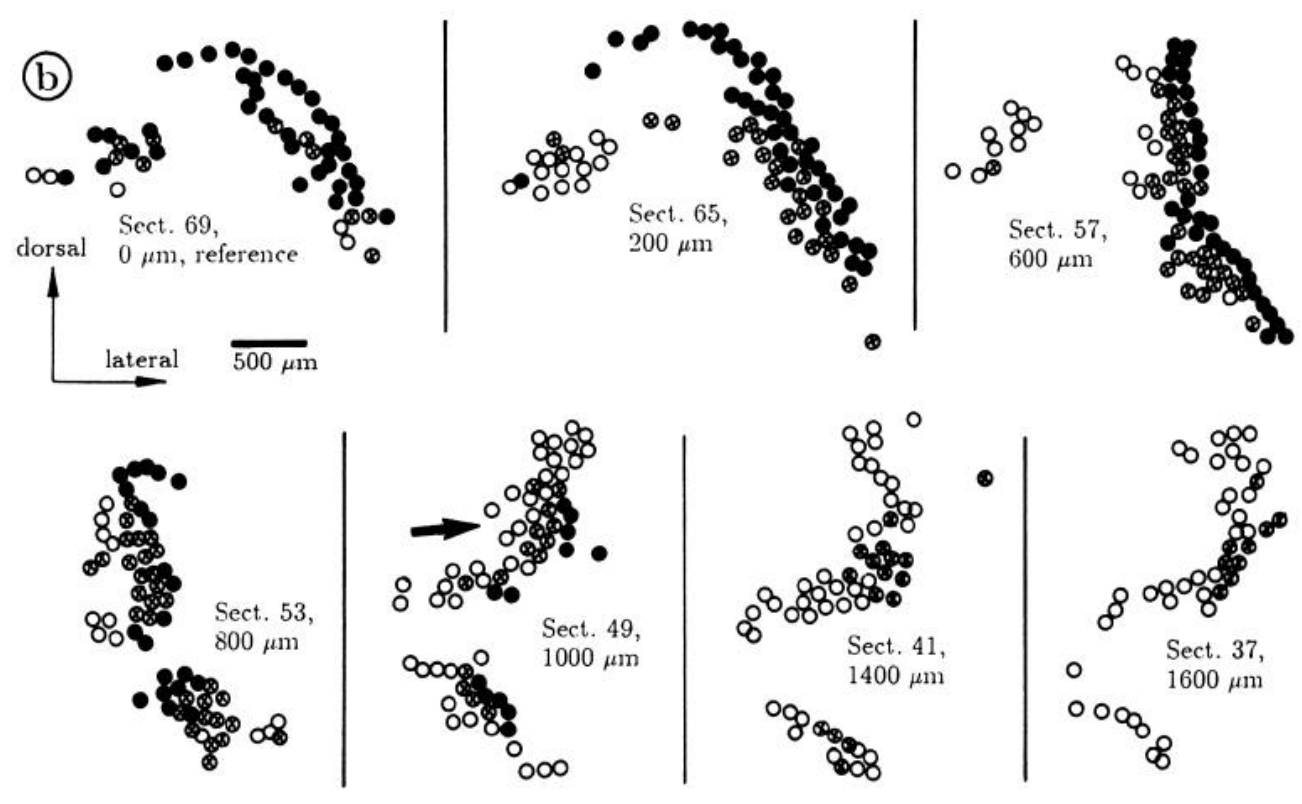

virtually no overlap between the differently labeled cell clusters in MGv occurs [e.g., di- and vi-cells in CAT033 (Fig. 3), CAT020 (Fig. 5), CAT026, CAT012.] Partial overlap in MGv was observed when the cortical injection sites were very close to each other (e.g., mi-cells and di-cells or mi-cells and vi-cells in CAT033). In all cases, however, the majority of cells labeled by a given tracer occur in regions free from cells labeled by other tracers.

\section{Topography of the medium-frequency projection $(3-8 \mathrm{kHz})$}

In experiment CAT016, $2 \mathrm{NY}$-injections were done into the dorsal part and $3 \mathrm{Bb}$-injections into the center and the ventral portion of the 4-6.7-kHz IFC (Fig. $6 a$ ). In LC, the di-cells (NY) form 2 separate groups: a rostrolateral array has the form of a narrow, vertically oriented band rostrally (Fig. $6 b$, sections 80 , 76) and diverges into two components further caudally (sections
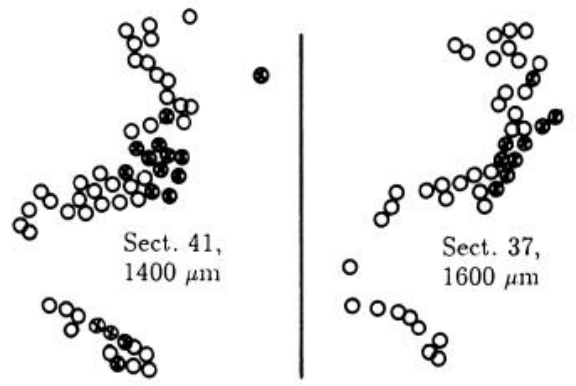

74-68). This column is marked by arrows " 1 " in Figure $6 b$. Caudoventromedially, a distinct band of di-cells appears (arrows " 2 " in Fig. $6 b$, which is oriented horizontally in sections 72-64 and folded lateralward further caudally (section 62). The vi-cells (Bb) are, in some sections, organized in 2 or 3 clusters, which are separated by gaps or groups of di-cells. However, all major components of the vi-array merge rostrally (section 74) and thus constitute a coherent population. Overlap between the differently labeled populations was minimal. Schematically, LC of CAT016 is shown in Figure $6 c$. There were only very few labeled cells in MC in this animal (not shown in Fig. 6, b, c).

In experiment CAT018, Bb was injected dorsally and NY ventrally into the 4-6.7-kHz IFC (Fig. 7a), as in CAT016. The results are schematically depicted in Figure $7 b$. Somewhat simplifying, one might say that the di-cells are wrapped around the vi-cells from the rostral, dorsal, ventral, and to a lesser extent, the medial aspects. Slight overlap between the differently labeled 


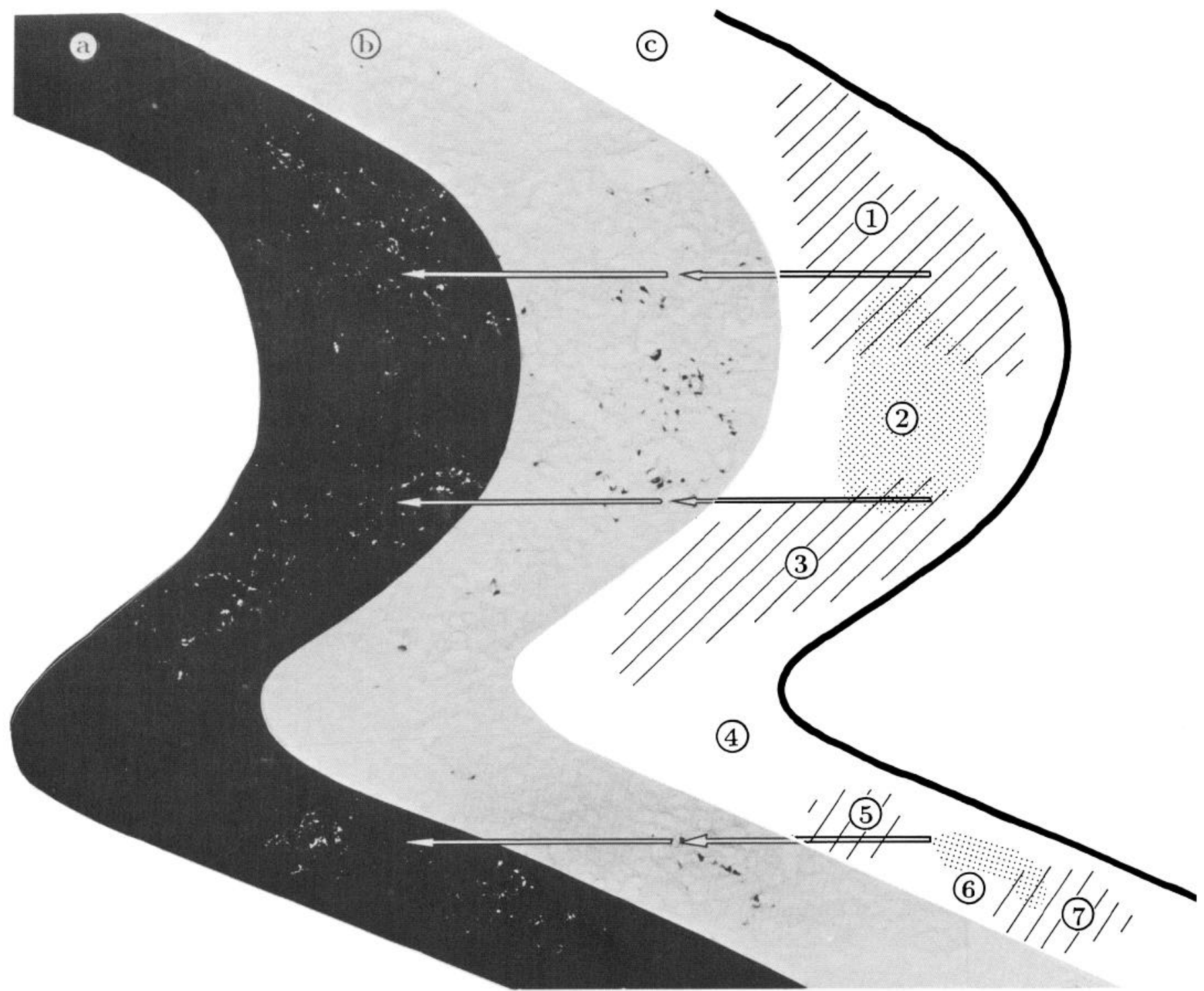

Figure 4. Experiment CAT033. $a$, Photograph of NY-labeled cells, from section 41 (frontal section, TMB-processed). A fluorescence filter with an excitation wavelength of $360 \mathrm{~nm}$ was used. $b$, Photograph of HRP-labeled cells, also from section 41 . $c$, Superimposed schematic drawing of $a$ and $b$. Areas containing NY-label are represented by shading, and the HRP-regions by stippling. For the meaning of the circled numbers (1-7) see text. Arrows indicate corresponding points in the figures.

populations, and also double labeling, was observed in some sections.

In experiment CAT017 (not illustrated), NY was injected dorsally (2 injections, spread over $1 \mathrm{~mm})$, HRP centrally $(3$ injections, spaced over ca. $1.3 \mathrm{~mm})$, and Bb ventrally ( 3 injections, spaced over $1.5 \mathrm{~mm}$ ) into the $4-6.7-\mathrm{kHz}$ IFC in AI. The distance between the NY-injections and the HRP-injection row was $0.5 \mathrm{~mm}$, and the distance between the HRP-injection row and the $\mathrm{Bb}$-injection row was $0.8 \mathrm{~mm}$. In LC, the di-cells (NY) are organized in 3 distinct bands, and the vi-cells in 2 bands. In location, these bands were roughly similar to the di- and vicells in CAT016 (Fig. 6). The mi-cells were located mainly in the center of LC (i.e., between the rostral and caudal poles of LC), immediately lateral to the di- and vi-cells. There was some overlap between the mi-cells and the other populations, but very little overlap between the di- and the vi-cells. The MC ofCAT017 consisted mainly of di-cells.
In 2 of the above-described cases, i.e., in CAT016 (Fig. 6) and CAT018 (Fig. 7), the cortical injection sites were similar in location; nevertheless, the structure of the projection differed in several respects. For example, in CAT016, the di-cells formed 2 distinct bands, while the di-cells in CAT018 were organized in 1 continuous band. Also, the differently labeled subbands interdigitated to a greater extent in CAT016 than in CAT018 (compare Figs. 6 and 7). In both animals, however, and in CAT017, the topography was similar in that the vi-cells were enclosed by di-cells rostrally, dorsally, and ventrally (or ventromedially).

\section{Discussion}

In this paper, a topographic order in the isofrequency dimension has been demonstrated in the anatomical projection from $\mathrm{MGv}$ to $\mathrm{AI}$ in cat. A limited sector $(1-2 \mathrm{~mm}$ in dorsoventral extent, in most experiments) of a cortical IFC receives input from 1-3 

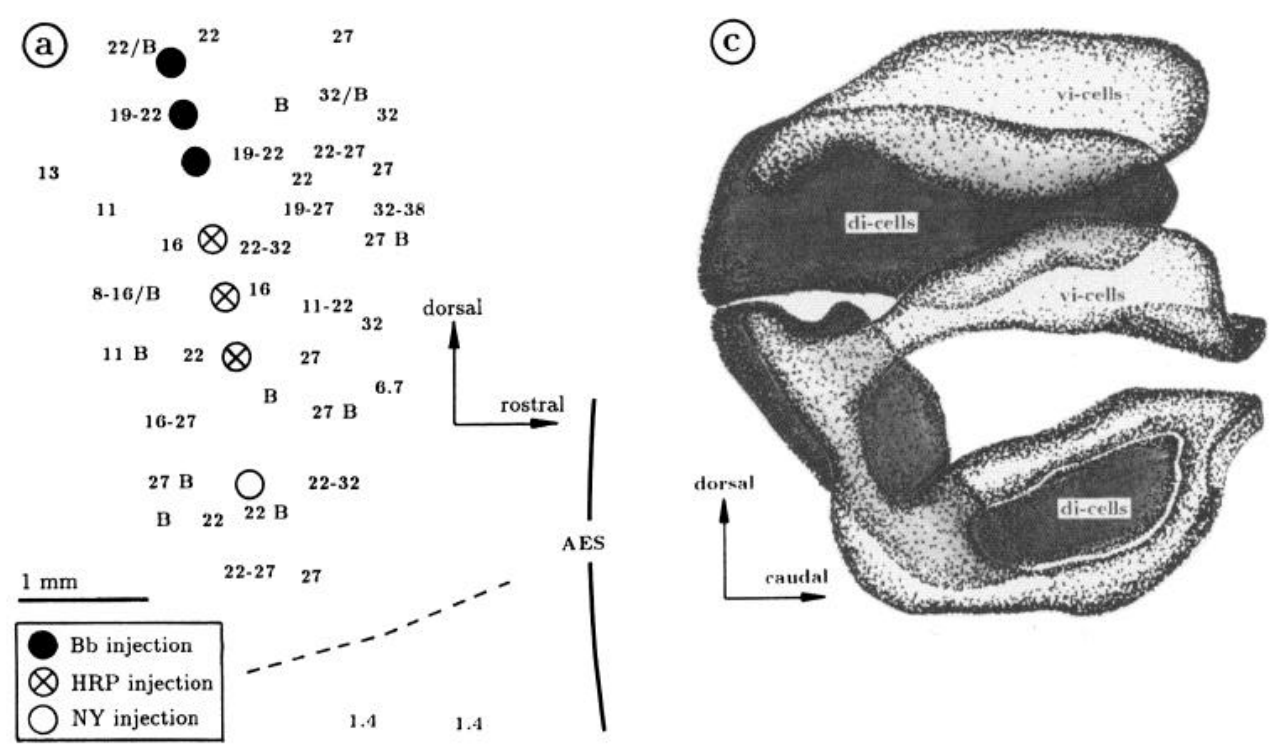

Figure 5. Experiment CAT020, $a$, BF map of field AI. Broken line represents the approximate ventral border of AI. $b$, Tracings of the labeled cells in LC (MC is not shown). $c$, Schematic drawing of LC in a parasagittal perspective, viewed laterally. See Figure 2 for further explanation.
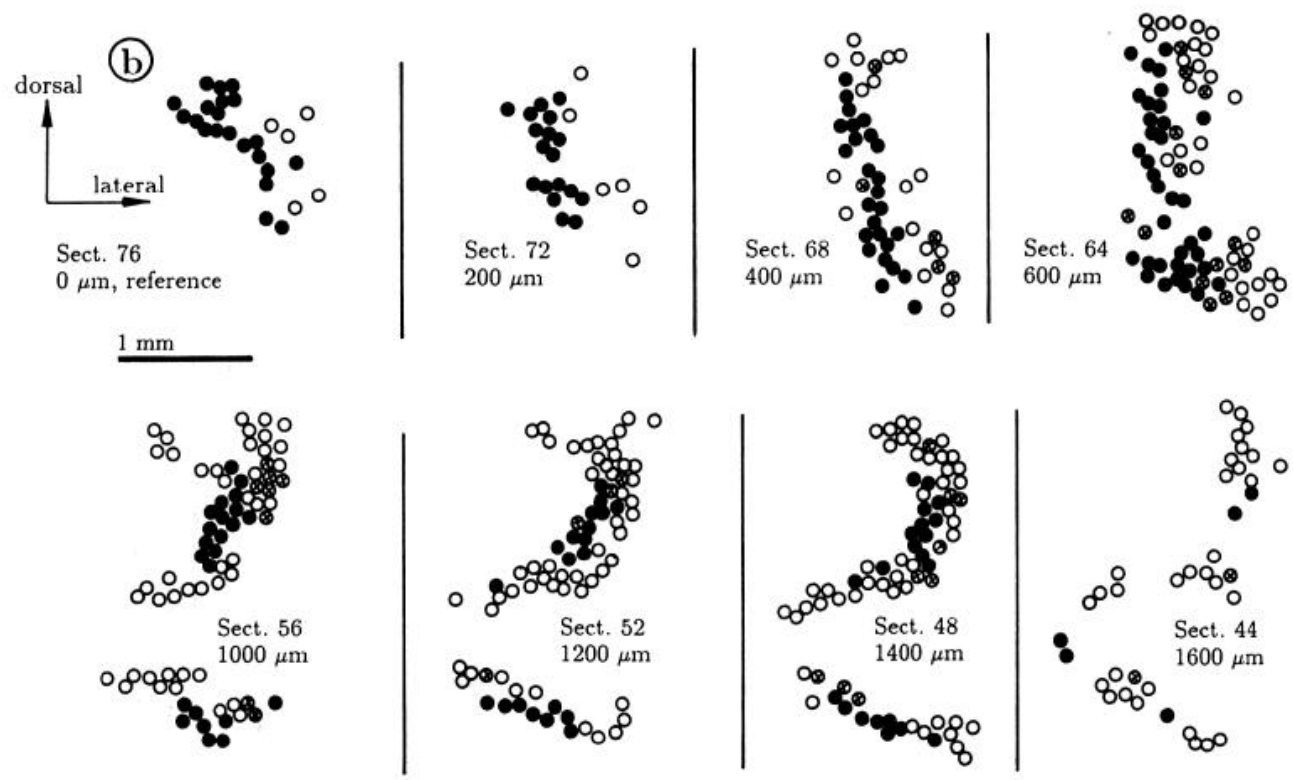

MGv neuronal arrays of variable shape. Other sectors of the same IFC are innervated by different arrays in MGv. The thalamic bands projecting to different parts of an IFC in AI may be folded into one another, and this accounts for the pattern of alternation of differently labeled clusters that is so striking in some of the frontal sections (e.g., CAT033, Figs. 3b, 4).

Consistently, a spatial gradient was found, in that after injections into the dorsal part of a cortical IFC, labeled cells were centered rostrally in $\mathrm{MGv}$, whereas ventral injections produced a labeled sheet centered further caudally. This rostrocaudal gradient can be more or less pronounced: For example, after dorsal injections, the labeled cells can be largely confined to the rostral half of LC, with almost no cells caudally (e.g., CAT033, Fig. $3 b$, di-cells); or, the sheet of di-cells extends through nearly the entire LC, but diminishes progressively in volume further caudally (e.g., CAT020, Fig. 5, b, c). The rostrocaudal gradient coexists with other gradients that are even more variable be- tween experiments (for example, compare rostral LC in CAT033, Fig. $3 b$, and in CAT020, Fig. $5 b$ ).

Experimental factors, for example variations in size and location of the injection sites, certainly contribute to the observed variability in topography. Differences between the individual brains may also exist. However, the relative importance of each of these possible factors cannot be deduced from our data. For this, a more precise mapping of field AI, including its binaural organization, and a larger number of experiments, would be necessary.

Topography appears similar for the medium- and high-frequency projections in essential features (e.g., rostrocaudal gradient, spatial separation of differently labeled cells), but there may be differences in the "local" gradients that coexist with the "global" rostrocaudal gradient. In the medium-frequency cases, the vi-cells are enclosed by di-cells rostrally, dorsally, and ventromedially. In the high-frequency cases, in rostral LC, we did 

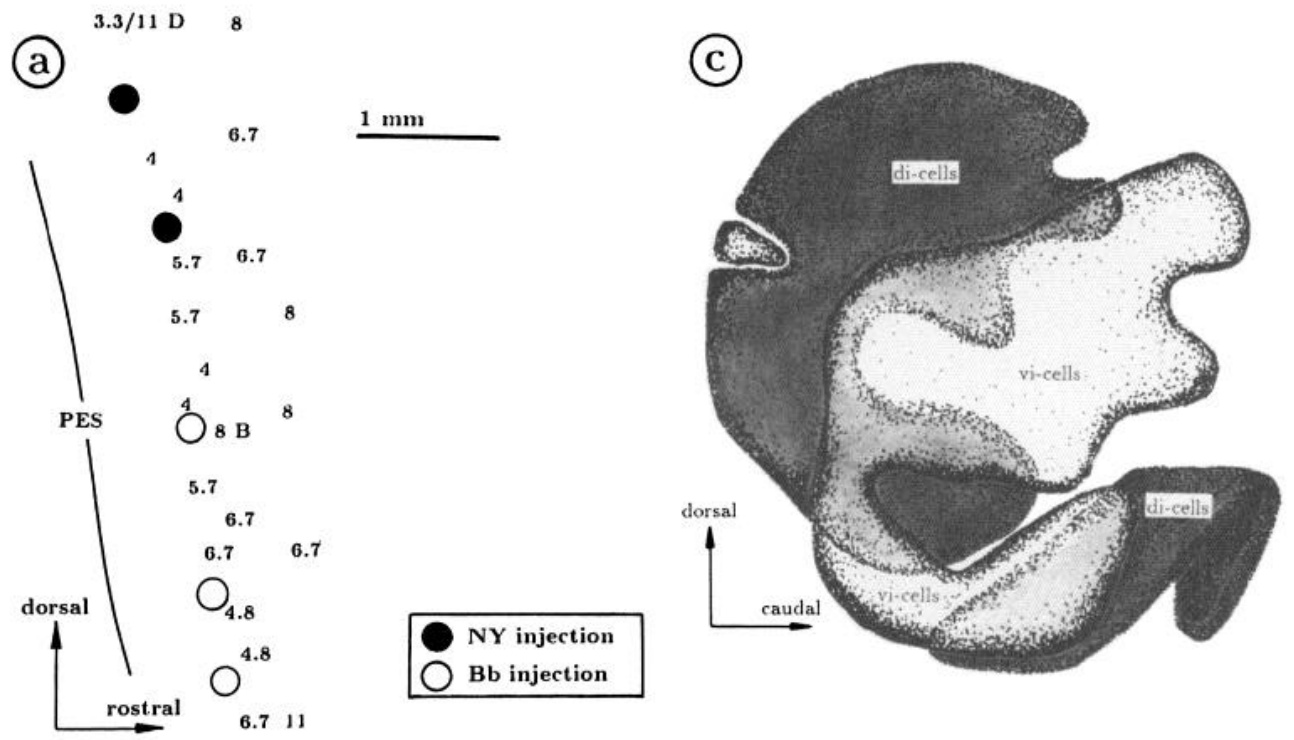

(b)
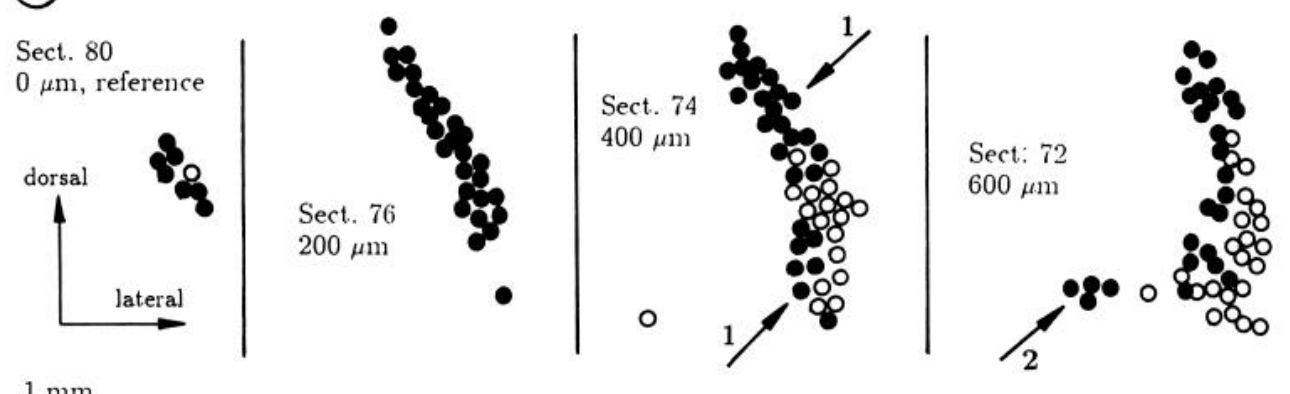

$1 \mathrm{~mm}$
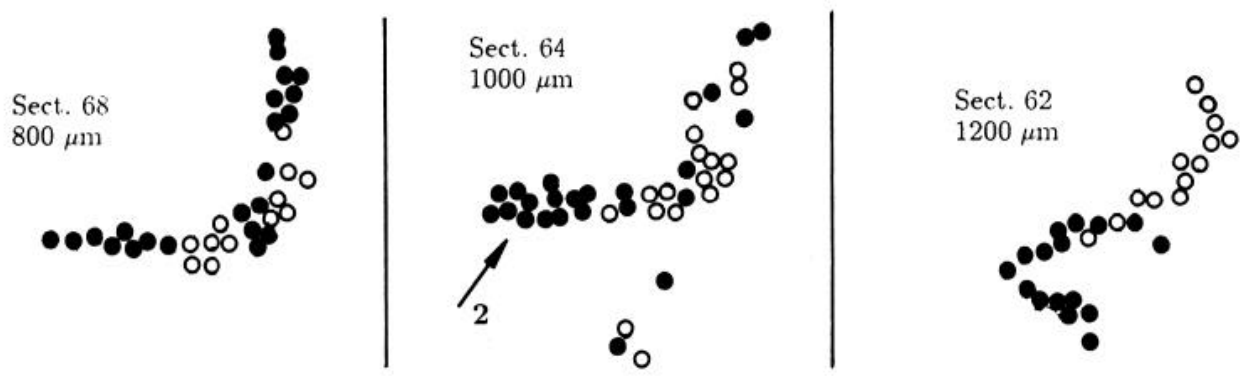

Figure 6. Experiment CAT016. $a, \mathrm{BF}$ map of field AI. $b$, Tracings of labeled cells in LC (MC is not shown). $c$, Schematic drawing of LC, in a parasagittal perspective, viewed laterally. See Figure 2 for further explanation. not observe this pattern, but, instead, a gradient from rostrolateral (di-cells) to caudomedial (mi- or vi-cells) (Fig. $3 b$ ), or a gradient in the opposite direction (Fig. $5 b$ ). Moreover, in LCd of the high-frequency cases, the di- or mi-cells are enclosed by vi-cells dorsally, ventromedially, and either laterally (Fig. $5 b$ ) or medially (Fig. $3 b$ ); this configuration, in turn, was not observed in any of the medium-frequency cases (compare Figs. $3 c$ and $5 c$ with $6 c$ and $7 b$ ). Thus, it is possible that topography in the isofrequency dimension depends on BF. Yet, variability between experiments with similar injections was high, and additional evidence is therefore required.

\section{The "divergence/convergence" pattern}

Current ideas on the structure of the auditory thalamocortical projection in the isofrequency dimension (Andersen et al., 1980;
Middlebrooks and Zook, 1983) are summarized in the introduction. The concept of a "divergence/convergence" architecture, proposed for cat (Fig. 1a), and the seeming difference between cat and guinea pig (Fig. $1 b$ ) were the main motivations for the present project.

Our results do not support the "divergence/convergence" concept.

1. In experiments CAT016 (Fig. 6), CAT017, CAT018 (Fig. 7), CAT020 (Fig. 5), and CAT033 (Figs. 3, 4), tracer injections spanned a strip of 4-5.5 mm; i.e., the entire (or nearly entire) dorsoventral extent of cortical IFCs was injected. Binaural bands measure about $0.5-1.5 \mathrm{~mm}$ in width (dorsoventral extent) and alternate along the cortical surface (Imig and Adrian, 1977; Middlebrooks et al., 1980; Middlebrooks and Zook, 1983). Therefore, it is highly probable that in each of the above listed 

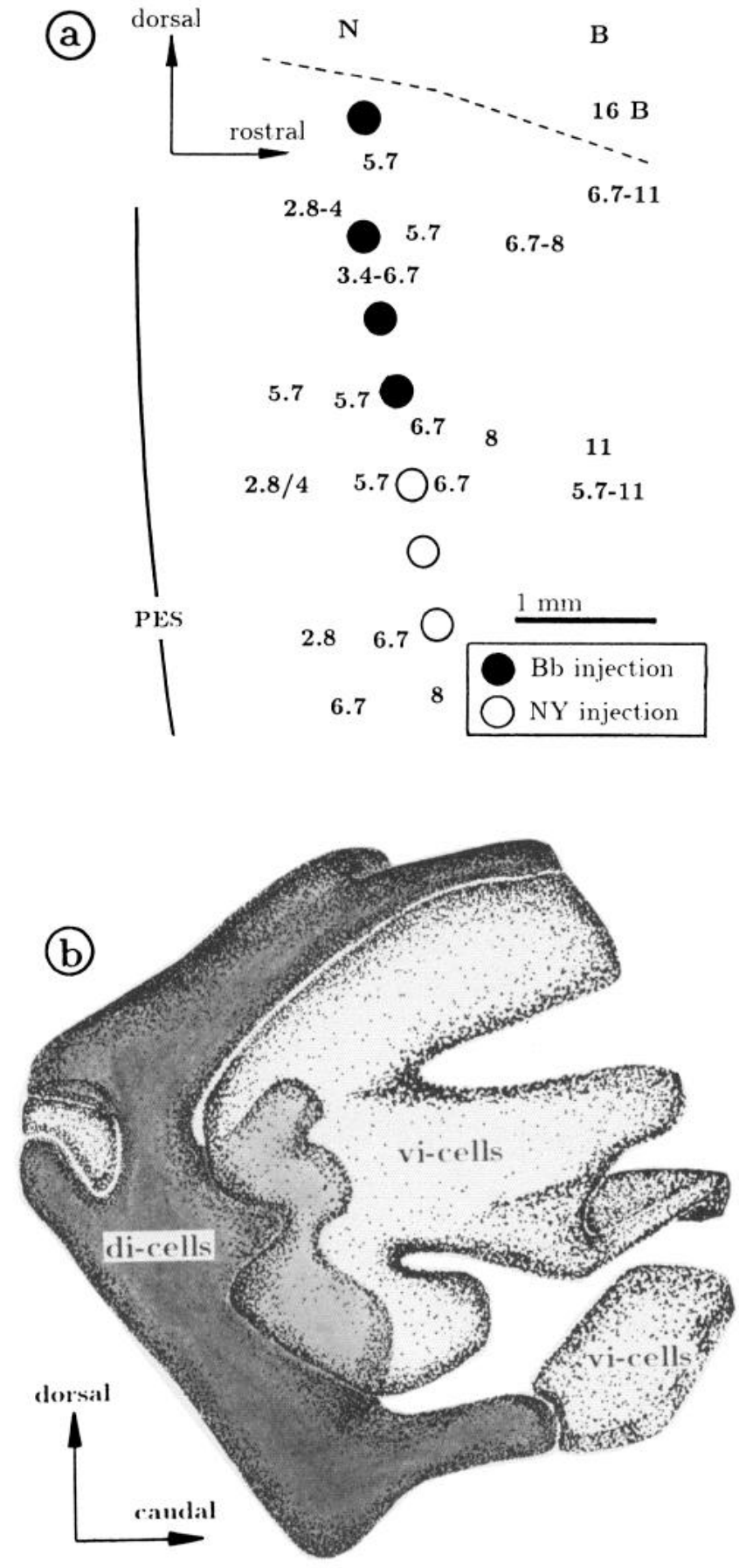

Figure 7. Experiment CAT018. $a$, BF map of field AI. The approximate dorsal border of $\mathrm{AI}$ is indicated by the broken line. $b$, Schematic drawing of LC, in a parasagittal perspective, viewed laterally. See Figure 2 for further explanation.

cases, different bands of the same binaural class-e.g., a ventral and a more dorsal EI-band-were injected by different tracers (see sketch in Fig. 8). If the EI-bands, or EE-bands, were innervated by coincident-or, in the case of EE-bands, largely coincident - thalamic populations, then the differently labeled thalamic arrays should, in these 5 experiments, overlap largely or completely. This was not the case.

2. In other experiments (CAT012, CAT026, CAT027), only parts of a cortical IFC were injected, with 2 different tracers (see Results). Slight overlap between differently labeled populations,

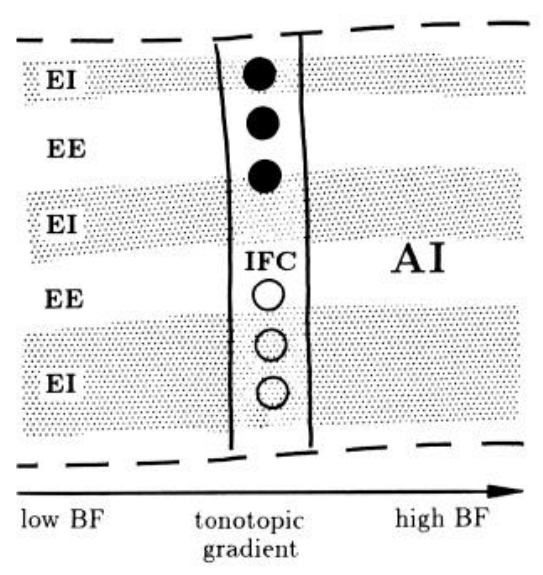

Figure 8. Schematic representation of binaural bands in field AI. The drawing illustrates a reasoning advanced in the text: If different tracers are positioned along the entire length of an IFC, then binaural strips of the same type are likely to be injected with different tracers. In the drawing, as an example, the ventral EI-band is injected with 1 tracer (open circles), and the dorsal EI-bands are injected with a different tracer (filled circles). The course of the binaural bands, which were not mapped in the present project, has been inferred from Middlebrooks and Zook (1983).

comparable to that in CAT033 (see Figs. 3, 4), was observed in one case (CAT027), whereas in the others, the spatial separation was nearly complete. These results are compatible with the "divergence/convergence" concept only under the assumption that in each experiment, by chance, the different injection sites coincided with different binaural bands, i.e., that the effective injection site of 1 tracer was located entirely within an EI-band, and that the other tracer was confined to an EE-band. But this is very improbable.

3. There are only 2 classes of binaural bands in AI. Consequently, if binaural bands of the same class were innervated by coincident thalamic populations, then, maximally, 2 differently labeled, spatially segregated populations in MGv could theoretically be found after different injections into a cortical IFC. But in CAT010 (Fig. 2), CAT033 (Fig. 3), CAT020 (Fig. 5), and CAT017, 3 different tracers were injected into single cortical IFCs, or over a large frequency range (overview experiments), and, in each case, 3 differently labeled, spatially segregated populations were found in MGv.

In summary, our results rule out the possibility that the "divergence/convergence" structure could exist in the projection from MGv to AI in cat, even in the absence of precise mapping of binaural bands in our experiments. The seeming substantial differences in the structure of the thalamocortical projection between cat and guinea pig (see beginning of this paper and Fig. 1), do not exist.

\section{Reasons for partial overlap}

The differently labeled populations in MGv did not intermingle when the injection sites of different tracers in AI were separated by more than 1.5 or $2 \mathrm{~mm}$ (measured from the centers of the injection sites); slight overlap at the borders of the differently labeled clusters in MGv was sometimes observed when different injection sites were closer to each other. This suggests that partial overlap depends on the distance between the injection sites.

Further interpretation of this finding is complicated by the difficulty of determining the effective injection site, as opposed to the apparent injection site, in studies using retrograde tracer substances (for discussion, see, e.g., Mesulam, 1982; Fahrbach 
et al., 1984). Therefore, in cases with nearby injection sites, it cannot be decided whether the partial overlap of differently labeled MGv clusters is due to overlap of the effective injection sites in the cortex or to other factors, for example a certain scatter, i.e., a limited divergence, in the thalamocortical projection.

Wc havc also performed experiments in which different tracers were injected into different cortical IFCs, to reveal tonotopy in MGv (Redies and Brandner, unpublished observation). In these experiments too, overlap of the differently labeled thalamic populations was observed when the different injection sites were nearby in the cortex, but not if they were distant. Thus, overlap is not a special feature of the structure of the projection in the isofrequency dimension, but exists also in the frequency dimension.

\section{Possible reasons for discrepancies}

Differences in the techniques used could account for the contradiction between our conclusions and the "divergence/convergence" concept. Andersen et al. had observed that single, moderately sized injections into AI produced a labeled sheet in MGv that is similar in extension to the sheet observed after larger or multiple injections into the same IFC. From this, they concluded that different parts of a cortical IFC must be innervated by coincident thalamic populations. The observation that arrays labeled by small injections often exhibit discontinuities, while arrays labeled by larger injections are continuous, has led to the hypothesis that the discontinuities could correspond to binaural bands (see introduction and Andersen et al., 1980; Merzenich et al., 1982). But the authors cited above have compared the thalamic populations labeled after single or multiple injections of tracer in different animals, and have not, as in the present project, compared thalamic arrays labeled by different tracers in the same animal.

This could possibly explain the contrary interpretations. Consider, for example, CAT020 and CAT033. The rostrocaudal extent of the vi-cells in CAT020 ( 1 injection, see Fig. 5) is nearly the same as the extent of the whole labeled sheet in CAT033 (9 injections, see Fig. 3). The main difference is indeed that the sheet of the vi-cells in CAT020 is discontinuous, while the labeled sheet (all tracers together) in CAT033 is continuous. Suppose we had performed a single injection of tracer in CAT020, in the position of the NY-injection, and multiple injections of 1 tracer in CAT033; then, in comparing both cases, we could have arrived at a similar, erroneous conclusion as described above! In other words, without simultaneous use of different tracers in the same animal, and if only a limited number of experiments is carried out, the topography in the isofrequency dimension may not become apparent.

Middlebrooks and Zook (1983, see introduction) injected different EI-bands also in different animals (i.e., injection into 1 EI-band per animal), and the above considerations may apply to their conclusions as well. The authors have, however, performed an experiment where 2 EE-bands were injected in the same animal. This particular case is consistent with our conclusion, since the arrays of differently labeled cells are differently centered (see Fig. 11 in Middlebrooks and Zook, 1983). There is more overlap between the different populations than we have observed in cases with equally distant injections $(2 \mathrm{~mm}$ between centers, see Fig. $1 b$ in Middlebrooks and Look, 1983), but this could be due to differential spread of the injected tracers in cortex: survival time was 50-60 hr in the study of Middlebrooks and Zook, which is twice as long as in the present project.

\section{References}

Andersen, R. A., P. L. Knight, and M. M. Merzenich (1980) The thalamocortical and corticothalamic connections of AI, AII and the anterior auditory field (AAF) in the cat: Evidence for two largely segregated systems of connections. J. Comp. Neurol. 194: 663-701.

Bentivoglio, M., H. G. J. M. Kuypers, C. E. Catsman-Berrevoets, H. Loewe, and O. Dann (1980) Two new fluorescent retrograde neuronal tracers which are transported over long distances. Neurosci. Lett. 18: 25-30.

Brugge, J. F. (1985) Patterns of organization in auditory cortex. J. Acoust. Soc. Am. 78: 353-359.

Brugge, J. F., and R. A. Reale (1985) Auditory cortex. In Cerebral Cortex, Volume 4: Association and Auditory Cortices, A. Peters and E. G. Jones, eds., pp. 229-272, Plenum, New York.

Fahrbach, S. E., J. I. Morrell, and D. W. Pfaff (1984) Temporal pattern of HRP spread from an iontophoretic deposit site and description of a new HRP-gel implant method. J. Comp. Neurol. 225: 605-619.

Imig, T. J., and H. O. Adrian (1977) Binaural columns in the primary field (AI) of cat auditory cortex. Brain Res. 138: 241-257.

Imig, T. J., and A. Morel (1983) Organization of the thalamocortical auditory system in the cat. Annu. Rev. Neurosci. 6: 95-120.

Imig, T. J., and A. Morel (1984) Topographic and cytoarchitectonic organization of thalamic neurons related to their targets in low-, middle-, and high-frequency representations in cat auditory cortex. $J$. Comp. Neurol. 227: 511-539.

Kuypers, H. G. J. M., M. Bentivoglio, D. van der Kooy, and C. E. Catsman-Berrevoets (1979) Retrograde transport of bisbenzimide and propidium iodide through axons to their parent cell bodies. Neurosci. Lett. 12: 1-7.

Kuypers, H. G. J. M., M. Bentivoglio, C. F. Catsman-Berrevoets, and A. T. Bharos (1980) Double retrograde neuronal labeling through divergent axon collaterals, using two fluorescent tracers with the same excitation wavelength which label different features of the cell. Exp. Brain Res. 40: 383-392.

Merzenich, M. M., S. A. Colwell, and R. A. Andersen (1982) Auditory forebrain organization. Thalamocortical and corticothalamic connections in cat. In Cortical Sensory Organization III, C. N. Woolsey, ed., pp. 43-57, Humana, Clifton, N.J.

Mesulam, M-M. (1982) Principles of horseradish peroxidase neurochemistry and their applications for tracing neural pathways - Axonal transport, enzyme histochemistry and light microscopic analysis. In Tracing Neural Connections with Horseradish Peroxidase, M-M. Mesulam, cd., pp. 1-152, Wiley, New York.

Middlebrooks, J. C., and J. M. Zook (1983) Intrinsic organization of the cat's medial geniculate body identified by projections to binaural response-specific bands in the primary auditory cortex. J. Neurosci. 3: 203-334.

Middlebrooks, J. C., R. W. Dykes, and M. M. Merzenich (1980) Binaural response specific bands in primary auditory cortex (AI) of the cat: Topographic organization orthogonal to isofrequency contours. Brain Res. 181: 31-48.

Morest, D. K. (1964) The neuronal architecture of the medial geniculate body of the cat. J. Anat. 98: 611-634.

Morest, D. K. (1965) The laminar structure of the medial geniculate body of the cat. J. Anat. 99: 143-160.

Mori, J., N. Hori, and N. Katsuda (1980) A new method for application of horscradish pcroxidase into a restricted area of the brain. Brain Res. Bull. 6: 19-22.

Phillips, D. P., and D. R. F. Irvine (1979) Methodological considerations in mapping auditory cortex: binaural columns in AI of cat. Brain Res. 161: 342-346.

Redies, H., U. Sieben, and O. D. Creutzfeldt (1989a) Functional subdivisions in the auditory cortex of the guinea pig. J. Comp. Neurol. 282: 473-488.

Redies, H., S. Brandner, and O. D. Creutzfeldt (1989b) Anatomy of the auditory thalamocortical system of the guinea pig. J. Comp. Neurol. 282: 489-511.

Suzuki, H., and M. Azuma (1976) A glass-insulated elgiloy microelectrode for recording unit activity in chronic monkey experiments. EEG Clin. Neurophysiol. 41: 93-95.

Wincr, J. A. (1985) The medial geniculate body of the cat. Adv. Anat. Embryol. Cell Biol. 86: 1-98. 